\title{
Common fixed point results under a new contractive condition without using continuity
}

\author{
Feng Gu' ${ }^{1}$ Yunjuan Shen ${ }^{1}$ and Lin Wang ${ }^{2 *}$ \\ Dedicated to Professor SS Chang on the occasion of his 80th birthday
}

"Correspondence:
WL64mail@aliyun.com
${ }^{2}$ College of Statistics and
Mathematics, Yunnan University of
Finance and Economics, Kunming,
Yunnan 650221, P.R. China
Full list of author information is
available at the end of the article

available at the end of the article

\begin{abstract}
In this paper, using the concept of the common (E.A) property, we prove a common fixed point theorem for a class of twice power type weakly compatible mappings in generalized metric space. Our results do not rely on any commuting or continuity condition of the mappings. We also state some examples to illustrate our new results in symmetric and nonsymmetric generalized metric spaces. It should be pointed out that this is the first time to use common (E.A) properties to discuss common fixed point problems of contractive mappings for twice power type in generalized metric spaces.
\end{abstract}

Keywords: generalized metric space; weakly compatible mappings; contraction mapping for twice power type; common (E.A) property

\section{Introduction and preliminaries}

In 2006, Mustafa and Sims [1] introduced a new structure of generalized metric space which is called a $G$-metric. Based on the notion of generalized metric spaces, Mustafa et al. [2-5], Obiedat and Mustafa [6], Aydi et al. [7, 8], Gajić and Stojaković [9], Zhou and $\mathrm{Gu}$ [10], Shatanawi [11] obtained some fixed point results for mappings satisfying different contractive conditions. Chugh et al. [12] obtained some fixed point results for maps satisfying property $P$ in $G$-metric spaces. The study of common fixed point problems in G-metric spaces was initiated by Abbas and Rhoades [13]. Subsequently, many authors obtained many common fixed point theorems for the mappings satisfying different contractive conditions; see [14-31] for more details. Recently, some authors using (E.A) property in generalized metric space to prove common fixed point, such as Abbas et al. [32], Mustafa et al. [33], Long et al. [34], Gu and Yin [35], Gu and Shatanawi [36].

Recently, Jleli and Samet [37] and Samet et al. [38] observed that some fixed point theorems in the context of a $G$-metric space can be proved (by simple transformation) using related existing results in the setting of a (quasi-) metric space. Namely, if the contraction condition of the fixed point theorem on G-metric space can be reduced to two variables, then one can construct an equivalent fixed point theorem in setting of usual metric space. This idea is not completely new, but it was not successfully used before (see [39]). 
Very recently, Karapınar and Agarwal suggest new contraction conditions in G-metric space in a way that the techniques in $[37,38]$ are not applicable. In this approach [40], contraction conditions cannot be expressed in two variables. So, in some cases, as is noticed even in Jleli-Samet's paper [37], when the contraction condition is of nonlinear type, this strategy cannot be always successfully used. This is exactly the case in our paper.

The purpose of this paper is to use the concept of the common (E.A) property and weakly compatible mappings to discuss common fixed point problem for a class of twice power type contractive mappings in the framework of a generalized metric space. Our results do not rely on any commuting or continuity condition of the mappings. We also state some examples to illustrate our new results in the framework of symmetric and nonsymmetric generalized metric spaces.

As far as we know, this is the first time to use common (E.A) properties to discuss common fixed point problems of contractive mappings for twice power type in generalized metric spaces.

Now we give preliminaries and basic definitions which are used throughout the paper.

Definition 1.1 [1] Let $X$ be a nonempty set, and let $G: X \times X \times X \rightarrow R^{+}$be a function satisfying the following axioms:

(G1) $G(x, y, z)=0$ if $x=y=z$;

(G2) $0<G(x, y, z)$ for all $x, y \in X$ with $x \neq y$;

(G3) $G(x, x, y) \leq G(x, y, z)$ for all $x, y, z \in X$ with $z \neq y$;

(G4) $G(x, y, z)=G(x, z, y)=G(y, z, x) \cdots$ (symmetry in all three variables);

(G5) $G(x, y, z) \leq G(x, a, a)+G(a, y, z)$ for all $x, y, z, a \in X$ (rectangle inequality).

Then the function $G$ is called a generalized metric or a $G$-metric on $X$, and the pair $(X, G)$ is called a $G$-metric space.

Definition 1.2 [1] Let $(X, G)$ be a $G$-metric space, and let $\left\{x_{n}\right\}$ a sequence of points in $X$, a point $x$ in $X$ is said to be the limit of the sequence $\left\{x_{n}\right\}, \lim _{n \rightarrow \infty} G\left(x, x_{n}, x_{m}\right)=0$, and one says that sequence $\left\{x_{n}\right\}$ is $G$-convergent to $x$.

Thus, if $x_{n} \rightarrow x$ or $\lim _{n \rightarrow \infty} x_{n}=x$ in a $G$-metric space $(X, G)$, then if for each $\epsilon>0$, there exists a positive integer $N$ such that $G\left(x, x_{n}, x_{m}\right)<\epsilon$ for all $n, m \geq N$.

Proposition 1.1 [1] Let $(X, G)$ be a G-metric space. Then the following are equivalent:

(1) $\left\{x_{n}\right\}$ is G-convergent to $x$.

(2) $G\left(x_{n}, x_{n}, x\right) \rightarrow 0$ as $n \rightarrow \infty$.

(3) $G\left(x_{n}, x, x\right) \rightarrow 0$ as $n \rightarrow \infty$.

(4) $G\left(x_{m}, x_{n}, x\right) \rightarrow 0$ as $m, n \rightarrow \infty$.

Definition 1.3 [1] Let $(X, G)$ be a $G$-metric space. A sequence $\left\{x_{n}\right\}$ is called $G$-Cauchy if, for each $\epsilon>0$, there exists a positive integer $N$ such that $G\left(x_{m}, x_{n}, x\right)<\epsilon$ for all $n, m, l \geq N$; i.e. if $G\left(x_{m}, x_{n}, x_{l}\right) \rightarrow 0$ as $m, n, l \rightarrow \infty$.

Proposition 1.2 [1] If $(X, G)$ is a G-metric space then the following are equivalent:

(1) The sequence $\left\{x_{n}\right\}$ is G-Cauchy.

(2) For each $\epsilon>0$, there exists a positive integer $N$ such that $G\left(x_{m}, x_{n}, x\right)<\epsilon$ for all $n, m, l \geq N$. 
Proposition 1.3 [1] Let $(X, G)$ be a G-metric space. Then the function $G(x, y, z)$ is jointly continuous in all three of its variables.

Definition 1.4 [1] A $G$-metric space $(X, G)$ is said to be $G$-complete if every $G$-Cauchy sequence in $(X, G)$ is $G$-convergent in $X$.

Definition 1.5 [41] Let $f$ and $g$ be self-maps of a set $X$. If $w=f x=g x$ for some $x$ in $X$, then $x$ is called a coincidence point of $f$ and $g$, and $w$ is called a point of coincidence of $f$ and $g$.

Definition 1.6 [41] Two self-mappings $f$ and $g$ on $X$ are said to be weakly compatible if they commute at coincidence points.

Definition 1.7 [32] Let $X$ be a G-metric space. Self-maps $f$ and $g$ on $X$ are said to satisfy the $G-(E . A)$ property if there exists a sequence $\left\{x_{n}\right\}$ in $X$ such that $\left\{x_{n}\right\}$ and $\left\{g x_{n}\right\}$ are $G$-convergent to some $t \in X$.

Definition 1.8 [32] Let $(X, d)$ be a $G$-metric space and $A, B, S$, and $T$ be four self-maps on $X$. The pairs $(A, S)$ and $(B, T)$ are said to satisfy the common $(E . A)$ property if there exist two sequences $\left\{x_{n}\right\}$ and $\left\{y_{n}\right\}$ in $X$ such that

$$
\lim _{n \rightarrow \infty} A x_{n}=\lim _{n \rightarrow \infty} S x_{n}=\lim _{n \rightarrow \infty} B y_{n}=\lim _{n \rightarrow \infty} T y_{n}=t
$$

for some $t \in X$.

Definition 1.9 [17] Self-mappings $f$ and $g$ of a $G$-metric space $(X, G)$ are said to be compatible if $\lim _{n \rightarrow \infty} G\left(f g x_{n}, g f x_{n}, g f x_{n}\right)=0$ and $\lim _{n \rightarrow \infty} G\left(g f x_{n}, f g x_{n}, f g x_{n}\right)=0$, whenever $\left\{x_{n}\right\}$ is a sequence in $X$ such that

$$
\lim _{n \rightarrow \infty} f x_{n}=\lim _{n \rightarrow \infty} g x_{n}=t
$$

for some $t \in X$.

\section{Main results}

Theorem 2.1 Let $(X, G)$ be a G-metric space. Suppose mappings $f, g, h, R, S, T: X \rightarrow X$ satisfying the following conditions:

$$
G^{2}(f x, g y, h z) \leq k \max \left\{\begin{array}{l}
G(R x, S y, T z) G(f x, R x, R x), \\
G(g y, S y, S y) G(h z, T z, T z), \\
G(f x, S y, T z) G(R x, g y, T z), \\
G(R x, S y, h z) G(f x, g y, T z), \\
G(f x, S y, h z) G(R x, g y, h z)
\end{array}\right\}
$$

for all $x, y, z \in X, 0 \leq k<1$. If one of the following conditions is satisfied, then the pairs $(f, R)$, $(g, S)$, and $(h, T)$ have a common point of coincidence in $X$.

(i) The subspace $R X$ is closed in $X, f X \subseteq S X, g X \subseteq T X$, and the two pairs of $(f, R)$ and $(g, S)$ satisfy the common (E.A) property.

(ii) The subspace $S X$ is closed in $X, g X \subseteq T X, h X \subseteq R X$, and the two pairs of $(g, S)$ and $(h, T)$ satisfy the common (E.A) property. 
(iii) The subspace $T X$ is closed in $X, f X \subseteq S X, h X \subseteq R X$, and the two pairs of $(f, R)$ and $(h, T)$ satisfy the common (E.A) property.

Further, if the pairs $(f, R),(g, S)$, and $(h, T)$ are weakly compatible, then $f, g, h, R, S$, and $T$ have a unique common fixed point in $X$.

Proof First we suppose that $R X$ is closed in $X, f X \subseteq S X, g X \subseteq T X$, and the two pairs of $(f, R)$ and $(g, S)$ satisfy the common $(E . A)$ property, then by Definition 1.8 we know that there exist two sequences $\left\{x_{n}\right\}$ and $\left\{y_{n}\right\}$ in $X$ such that

$$
\lim _{n \rightarrow \infty} f x_{n}=\lim _{n \rightarrow \infty} R x_{n}=\lim _{n \rightarrow \infty} g y_{n}=\lim _{n \rightarrow \infty} S y_{n}=t
$$

for some $t \in X$.

Since $g X \subseteq T X$, there exists a sequence $\left\{z_{n}\right\}$ in $X$ such that $g y_{n}=T z_{n}$. So we get $\lim _{n \rightarrow \infty} T z_{n}=\lim _{n \rightarrow \infty} g y_{n}=t$. By the condition (2.1) we have

$$
G^{2}\left(f x_{n}, g y_{n}, h z_{n}\right) \leq k \max \left\{\begin{array}{l}
G\left(R x_{n}, S y_{n}, T z_{n}\right) G\left(f x_{n}, R x_{n}, R x_{n}\right), \\
G\left(g y_{n}, S y_{n}, S y_{n}\right) G\left(h z_{n}, T z_{n}, T z_{n}\right), \\
G\left(f x_{n}, S y_{n}, T z_{n}\right) G\left(R x_{n}, g y_{n}, T z_{n}\right), \\
G\left(R x_{n}, S y_{n}, h z_{n}\right) G\left(f x_{n}, g y_{n}, T z_{n}\right), \\
G\left(f x_{n}, S y_{n}, h z_{n}\right) G\left(R x_{n}, g y_{n}, h z_{n}\right)
\end{array}\right\} .
$$

Letting $n \rightarrow \infty$, we have

$$
G^{2}\left(t, t, \lim _{n \rightarrow \infty} h z_{n}\right) \leq k G^{2}\left(t, t, \lim _{n \rightarrow \infty} h z_{n}\right)
$$

this gives $G^{2}\left(t, t, \lim _{n \rightarrow \infty} h z_{n}\right)=0$, since $0 \leq k<1$. Hence $\lim _{n \rightarrow \infty} h z_{n}=t$.

Since $R X$ is a closed subspace of $X$, and $\lim _{n \rightarrow \infty} R x_{n}=t$, there exists a point $u \in X$ such that $R u=t$. By the condition (2.1) we have

$$
G^{2}\left(f u, g y_{n}, h z_{n}\right) \leq k \max \left\{\begin{array}{c}
G\left(R u, S y_{n}, T z_{n}\right) G(f u, R u, R u), \\
G\left(g y_{n}, S y_{n}, S y_{n}\right) G\left(h z_{n}, T z_{n}, T z_{n}\right), \\
G\left(f u, S y_{n}, T z_{n}\right) G\left(R u, g y_{n}, T z_{n}\right), \\
G\left(R u, S y_{n}, h z_{n}\right) G\left(f u, g y_{n}, T z_{n}\right), \\
G\left(f u, S y_{n}, h z_{n}\right) G\left(R u, g y_{n}, h z_{n}\right)
\end{array}\right\} .
$$

Letting $n \rightarrow \infty$, we have $G^{2}(f u, t, t) \leq 0$, hence $f u=t$. Thus $R u=f u=t$, so $u$ is the coincidence point of the pair $(f, R)$.

Since $f X \subseteq S X$ and $f u=t$, there exists a point $v \in X$ such that $S v=f u=t$. By the condition (2.1) we have

$$
G^{2}\left(f u, g v, h z_{n}\right) \leq k \max \left\{\begin{array}{c}
G\left(R u, S v, T z_{n}\right) G(f u, R u, R u), \\
G(g v, S v, S v) G\left(h z_{n}, T z_{n}, T z_{n}\right), \\
G\left(f u, S v, T z_{n}\right) G\left(R u, g v, T z_{n}\right), \\
G\left(R u, S v, h z_{n}\right) G\left(f u, g v, T z_{n}\right), \\
G\left(f u, S v, h z_{n}\right) G\left(R u, g v, h z_{n}\right)
\end{array}\right\} .
$$

Letting $n \rightarrow \infty$, we have $G^{2}(t, g v, t) \leq 0$, hence $g v=t$. Thus $S v=g v=t$, so $v$ is the coincidence point of the pair $(g, S)$. 
Since $g X \subseteq T X$ and $g v=t$, there exists a point $w \in X$ such that $T w=g v=t$. By the condition (2.1) we have

$$
\begin{aligned}
G^{2}(t, t, h w) & =G^{2}(f u, g v, h w) \\
& \leq k \max \left\{\begin{array}{l}
G(R u, S v, T w) G(f u, R u, R u), \\
G(g v, S v, S v) G(h w, T w, T w), \\
G(f u, S v, T w) G(R u, g v, T w), \\
G(R u, S v, h w) G(f u, g v, T w), \\
G(f u, S v, h w) G(R u, g v, h w)
\end{array}\right\} \\
& =k G^{2}(t, t, h w),
\end{aligned}
$$

hence $h w=t$, since $0 \leq k<1$. Thus $T w=h w=t$, so $w$ is the coincidence point of the pair $(h, T)$.

In the above proof we get $f u=R u=g v=S v=h w=T w=t$. Then we get $f t=R t, g t=S t$, and $h t=T t$, since the pairs $(f, R),(g, S)$, and $(h, T)$ are weakly compatible. By the condition (2.1), we have

$$
\begin{aligned}
G^{2}(f t, t, t) & =G^{2}(f t, g v, h w) \\
& \leq k \max \left\{\begin{array}{c}
G(R t, S v, T w) G(f t, R t, R t), \\
G(g v, S v, S v) G(h w, T w, T w), \\
G(f t, S v, T w) G(R t, g v, T w), \\
G(R t, S v, h w) G(f t, g v, T w), \\
G(f t, S v, h w) G(R t, g v, h w)
\end{array}\right\} \\
& =k G^{2}(f t, t, t),
\end{aligned}
$$

hence $G^{2}(f t, t, t)=0$, since $0 \leq k<1$. Thus $f t=t=R t$. Similarly, it can be shown that $g t=$ $S t=t$ and $h t=T t=t$, which means that $t$ is a common fixed point of $f, g, h, R, S$, and $T$.

Now we prove the uniqueness of the common fixed point $t$.

Let $t$ and $p$ be two common fixed point of $f, g, h, R, S$, and $T$, then using the condition (2.1), we have

$$
\begin{aligned}
G^{2}(p, t, t) & =G^{2}(f p, g t, h t) \\
& \leq k \max \left\{\begin{array}{c}
G(R p, S t, T t) G(f p, R p, R p), \\
G(g t, S t, S t) G(h t, T t, T t), \\
G(f p, S t, T t) G(R p, g t, T t), \\
G(R p, S t, h t) G(f p, g t, T t), \\
G(f p, S t, h t) G(R p, g t, h t)
\end{array}\right\} \\
& =k G^{2}(p, t, t),
\end{aligned}
$$

hence $G^{2}(p, t, t)=0$, since $0 \leq k<1$. Thus $p=t$. So common fixed point is unique.

Example 2.1 Let $X=[0,1]$ be a $G$-metric space with

$$
G(x, y, z)=|x-y|+|y-z|+|z-x| .
$$


We define mappings $f, g, h, R, S$, and $T$ on $X$ by

$$
\begin{array}{cl}
f x=\left\{\begin{array}{ll}
1, & x \in\left[0, \frac{1}{2}\right], \\
\frac{4}{5}, & x \in\left(\frac{1}{2}, 1\right],
\end{array} \quad g x=\left\{\begin{array}{ll}
\frac{5}{6}, & x \in\left[0, \frac{1}{2}\right], \\
\frac{4}{5}, & x \in\left(\frac{1}{2}, 1\right],
\end{array} \quad h x= \begin{cases}\frac{6}{7}, & x \in\left[0, \frac{1}{2}\right], \\
\frac{4}{5}, & x \in\left(\frac{1}{2}, 1\right],\end{cases} \right.\right. \\
R x=\left\{\begin{array}{lll}
0, & x \in\left[0, \frac{1}{2}\right], \\
\frac{4}{5}, & x \in\left(\frac{1}{2}, 1\right), \\
\frac{6}{7}, & x=1,
\end{array} \quad S x=\left\{\begin{array}{ll}
1, & x \in\left[0, \frac{1}{2}\right] \\
\frac{4}{5}, & x \in\left(\frac{1}{2}, 1\right), \\
0, & x=1,
\end{array} \quad T x= \begin{cases}0, & x \in\left[0, \frac{1}{2}\right], \\
\frac{4}{5}, & x \in\left(\frac{1}{2}, 1\right), \\
\frac{5}{6}, & x=1 .\end{cases} \right.\right.
\end{array}
$$

Clearly, from the above functions we know that the subspace $R X$ is closed in $X, f X \subseteq S X$, $g X \subseteq T X, h X \subseteq R X$ and the pairs $(f, R),(g, S),(h, T)$ be weakly compatible. The pairs $(f, R)$ and $(g, S)$ satisfy the common (E.A) property, let $x_{n}=\frac{6}{7}$ and $y_{n}=\frac{5}{6}$ for each $n \in N$ be the required sequences.

Now we prove that the mappings $f, g, h, R, S$, and $T$ are satisfying the condition (2.1) of Theorem 2.1 with $k=\frac{1}{4} \in[0,1)$. Let

$$
M(x, y, z)=\max \left\{\begin{array}{l}
G(R x, S y, T z) G(f x, R x, R x), \\
G(g y, S y, S y) G(h z, T z, T z), \\
G(f x, S y, T z) G(R x, g y, T z), \\
G(R x, S y, h z) G(f x, g y, T z), \\
G(f x, S y, h z) G(R x, g y, h z)
\end{array}\right\} .
$$

Case (1) If $x, y, z \in\left[0, \frac{1}{2}\right]$, then we have

$$
\begin{aligned}
& G^{2}(f x, g y, h z)=G^{2}\left(1, \frac{5}{6}, \frac{6}{7}\right)=\frac{1}{9}, \\
& G(R x, S y, T z) G(f x, R x, R x)=G(0,1,0) G(1,0,0)=4 .
\end{aligned}
$$

Thus we have

$$
G^{2}(f x, g y, h z)=\frac{1}{9}<\frac{1}{4} \cdot 4=k G(R x, S y, T z) G(f x, R x, R x) \leq k M(x, y, z) .
$$

Case (2) If $x, y \in\left[0, \frac{1}{2}\right], z \in\left(\frac{1}{2}, 1\right]$, then we have

$$
G^{2}(f x, g y, h z)=G^{2}\left(1, \frac{5}{6}, \frac{4}{5}\right)=\frac{4}{25} .
$$

If $z=1$, then

$$
G(R x, S y, T z) G(f x, R x, R x)=G\left(0,1, \frac{5}{6}\right) G(1,0,0)=4
$$

If $z \in\left(\frac{1}{2}, 1\right)$, then

$$
G(R x, S y, T z) G(f x, R x, R x)=G\left(0,1, \frac{4}{5}\right) G(1,0,0)=4
$$


So we know $G(R x, S y, T z) G(f x, R x, R x)=4$. Thus we have

$$
G^{2}(f x, g y, h z)=\frac{4}{25}<\frac{1}{4} \cdot 4=k G(R x, S y, T z) G(f x, R x, R x) \leq k M(x, y, z) .
$$

Case (3) If $x, z \in\left[0, \frac{1}{2}\right], y \in\left(\frac{1}{2}, 1\right]$, then we have

$$
G^{2}(f x, g y, h z)=G^{2}\left(1, \frac{4}{5}, \frac{6}{7}\right)=\frac{4}{25} .
$$

If $y=1$, then

$$
G(f x, S y, T z) G(R x, g y, T z)=G(1,0,0) G\left(0, \frac{4}{5}, 0\right)=\frac{16}{5} .
$$

If $y \in\left(\frac{1}{2}, 1\right)$, then

$$
G(f x, S y, T z) G(R x, g y, T z)=G\left(1, \frac{4}{5}, 0\right) G\left(0, \frac{4}{5}, 0\right)=\frac{16}{5} .
$$

So we know $G(R x, S y, T z) G(f x, R x, R x)=\frac{16}{5}$. Thus we have

$$
G^{2}(f x, g y, h z)=\frac{4}{25}<\frac{1}{4} \cdot \frac{16}{5}=k G(f x, S y, T z) G(R x, g y, T z) \leq k M(x, y, z)
$$

Case (4) If $y, z \in\left[0, \frac{1}{2}\right], x \in\left(\frac{1}{2}, 1\right]$, then we have

$$
G^{2}(f x, g y, h z)=G^{2}\left(\frac{4}{5}, \frac{5}{6}, \frac{6}{7}\right)=\frac{16}{1,225} .
$$

If $x=1$, then

$$
G(f x, S y, T z) G(R x, g y, T z)=G\left(\frac{4}{5}, 1,0\right) G\left(\frac{6}{7}, \frac{5}{6}, 0\right)=\frac{24}{7} .
$$

If $x \in\left(\frac{1}{2}, 1\right)$, then

$$
G(f x, S y, T z) G(R x, g y, T z)=G\left(\frac{4}{5}, 1,0\right) G\left(\frac{4}{5}, \frac{5}{6}, 0\right)=\frac{10}{3} .
$$

So we know $G(f x, S y, T z) G(R x, g y, T z) \geq \frac{10}{3}$. Thus we have

$$
G^{2}(f x, g y, h z)=\frac{16}{1,225}<\frac{1}{4} \cdot \frac{10}{3} \leq k G(f x, S y, T z) G(R x, g y, T z) \leq k M(x, y, z) .
$$

Case (5) $x \in\left[0, \frac{1}{2}\right], y, z \in\left(\frac{1}{2}, 1\right]$, then we have

$$
G^{2}(f x, g y, h z)=G^{2}\left(1, \frac{4}{5}, \frac{4}{5}\right)=\frac{4}{25} \text {. }
$$


If $y=1$, then

$$
G(f x, S y, h z) G(R x, g y, h z)=G\left(1,0, \frac{4}{5}\right) G\left(0, \frac{4}{5}, \frac{4}{5}\right)=\frac{16}{5} .
$$

If $y \in\left(\frac{1}{2}, 1\right)$, then

$$
G(f x, S y, h z) G(R x, g y, h z)=G\left(1, \frac{4}{5}, \frac{4}{5}\right) G\left(0, \frac{4}{5}, \frac{4}{5}\right)=\frac{16}{25}
$$

So we know $G(f x, S y, h z) G(R x, g y, h z) \geq \frac{16}{25}$. Thus we have

$$
G^{2}(f x, g y, h z)=\frac{4}{25}=\frac{1}{4} \cdot \frac{16}{25} \leq k G(f x, S y, h z) G(R x, g y, h z) \leq k M(x, y, z) .
$$

Case (6) $y \in\left[0, \frac{1}{2}\right], x, z \in\left(\frac{1}{2}, 1\right]$, then we have

$$
G^{2}(f x, g y, h z)=G^{2}\left(\frac{4}{5}, \frac{5}{6}, \frac{4}{5}\right)=\frac{1}{225} .
$$

If $x=1$, then

$$
G(f x, S y, h z) G(R x, g y, h z)=G\left(\frac{4}{5}, 1, \frac{4}{5}\right) G\left(\frac{6}{7}, \frac{5}{6}, \frac{4}{5}\right)=\frac{8}{175} \text {. }
$$

If $x \in\left(\frac{1}{2}, 1\right)$, then

$$
G(f x, S y, h z) G(R x, g y, h z)=G\left(\frac{4}{5}, 1, \frac{4}{5}\right) G\left(\frac{4}{5}, \frac{5}{6}, \frac{4}{5}\right)=\frac{2}{75} .
$$

So we know $G(f x, S y, h z) G(R x, g y, h z) \geq \frac{2}{75}$. Thus we have

$$
G^{2}(f x, g y, h z)=\frac{1}{225}<\frac{1}{4} \cdot \frac{2}{75} \leq k G(f x, S y, h z) G(R x, g y, h z) \leq k M(x, y, z) .
$$

Case (7) $z \in\left[0, \frac{1}{2}\right], x, y \in\left(\frac{1}{2}, 1\right]$, then we have

$$
G^{2}(f x, g y, h z)=G^{2}\left(\frac{4}{5}, \frac{4}{5}, \frac{6}{7}\right)=\frac{16}{1,225} .
$$

If $x=1, y=1$, then

$$
G(f x, S y, T z) G(R x, g y, T z)=G\left(\frac{4}{5}, 0,0\right) G\left(\frac{6}{7}, \frac{4}{5}, 0\right)=\frac{96}{35} .
$$

If $x=1, y \in\left(\frac{1}{2}, 1\right)$, then

$$
G(f x, S y, T z) G(R x, g y, T z)=G\left(\frac{4}{5}, \frac{4}{5}, 0\right) G\left(\frac{6}{7}, \frac{4}{5}, 0\right)=\frac{96}{35} .
$$


If $y=1, x \in\left(\frac{1}{2}, 1\right)$, then

$$
G(f x, S y, T z) G(R x, g y, T z)=G\left(\frac{4}{5}, 0,0\right) G\left(\frac{4}{5}, \frac{4}{5}, 0\right)=\frac{64}{25} .
$$

If $x, y \in\left(\frac{1}{2}, 1\right)$, then

$$
G(f x, S y, T z) G(R x, g y, T z)=G\left(\frac{4}{5}, \frac{4}{5}, 0\right) G\left(\frac{4}{5}, \frac{4}{5}, 0\right)=\frac{64}{25} .
$$

So we know $G(f x, S y, T z) G(R x, g y, T z) \geq \frac{64}{25}$. Thus we have

$$
G^{2}(f x, g y, h z)=\frac{16}{1,225}<\frac{1}{4} \cdot \frac{64}{25} \leq k G(f x, S y, T z) G(R x, g y, T z) \leq k M(x, y, z) .
$$

Case (8) If $x, y, z \in\left(\frac{1}{2}, 1\right]$, then

$$
G^{2}(f x, g y, h z)=G^{2}\left(\frac{4}{5}, \frac{4}{5}, \frac{4}{5}\right)=0 \leq \frac{1}{4} M(x, y, z)=k M(x, y, z)
$$

Then in all the above cases, the mappings $f, g, h, R, T$, and $S$ are satisfying the condition (2.1) of Theorem 2.1 with $k=\frac{1}{4}$, so that all the conditions of Theorem 2.1 are satisfied. Moreover, $\frac{4}{5}$ is the unique common fixed point of $f, g, h, R, T$, and $S$.

The following example supports the usability of our results for nonsymmetric generalized metric spaces.

Example 2.2 Let $X=\{0,1,2\}$ be a set with $G$-metric defined by Table 1 . It is easy to see that $(X, G)$ is a nonsymmetric generalized metric space. Let the maps $f, g, h, R, S, T: X \rightarrow X$ be defined by Table 2 .

Clearly, the subspace $R X, S X$, and $T X$ are closed in $X, f X \subseteq S X, g X \subseteq T X$, and $h X \subseteq R X$ with the pairs $(f, R),(g, S)$, and $(h, T)$ being weakly compatible. Also two pairs $(f, R)$ and $(g, S)$ satisfy the common $(E . A)$ property, indeed, $x_{n}=0$ and $y_{n}=1$ for each $n \in \mathbb{N}$ are the required sequences.

To check the contractive condition (2.1) for all $x, y \in X$, we consider the following cases.

Table 1 The definition of $G$-metric on $X$

\begin{tabular}{ll}
\hline $\boldsymbol{( x , y , z )}$ & $\boldsymbol{G}(\boldsymbol{x}, \boldsymbol{y}, \boldsymbol{z})$ \\
\hline$(0,0,0),(1,1,1),(2,2,2)$ & 0 \\
$(0,0,1),(0,1,0),(1,0,0),(0,1,1),(1,0,1),(1,1,0)$ & 1 \\
$(1,2,2),(2,1,2),(2,2,1)$ & 2 \\
$(0,0,2),(0,2,0),(2,0,0),(0,2,2),(2,0,2),(2,2,0)$ & 3 \\
$(1,1,2),(1,2,1),(2,1,1),(0,1,2),(0,2,1),(1,0,2),(1,2,0),(2,0,1),(2,1,0)$ & 4 \\
\hline
\end{tabular}

Table 2 The definition of maps $f, g, h, R, S$ and $T$ on $X$

\begin{tabular}{lllllll}
\hline $\boldsymbol{x}$ & $\boldsymbol{f ( x )}$ & $\boldsymbol{g ( x )}$ & $\boldsymbol{h}(\boldsymbol{x})$ & $\boldsymbol{R}(\boldsymbol{x})$ & $\boldsymbol{S}(\boldsymbol{x})$ & $\boldsymbol{T}(\boldsymbol{x})$ \\
\hline 0 & 0 & 0 & 0 & 0 & 0 & 0 \\
1 & 0 & 0 & 1 & 1 & 0 & 2 \\
2 & 0 & 1 & 0 & 1 & 2 & 1 \\
\hline
\end{tabular}


Note that for Case (1) $x=y=z=0$, (2) $x=y=0, z=2$, (3) $x=z=0, y=1$, (4) $x=0$, $y=1, z=2$, (5) $x=1, y=z=0$, (6) $x=1, y=0, z=2$, (7) $x=y=1, z=0$, (8) $x=y=1, z=2$, (9) $x=2, y=z=0,(10) x=z=2, y=0$, (11) $x=2, y=1, z=0$, and (12) $x=z=2, y=1$.

We have $G(f x, g y, h z)=G(0,0,0)=0$, and hence (2.1) is obviously satisfied.

Case (13) If $x=y=0, z=1$, then $f x=g y=0, h z=1, R x=S y=0, T z=2$, hence we have

$$
\begin{aligned}
G^{2}(f 0, g 0, h 1) & =G^{2}(0,0,1)=1 \\
& <\frac{1}{3} \times 9=\frac{1}{3} G(0,0,2) G(0,0,2) \\
& =\frac{1}{3} G(f 0, S 0, T 1) G(R 0, g 0, T 1) \\
& \leq \frac{1}{3} M(0,0,1) .
\end{aligned}
$$

Case (14) If $x=0, y=z=1$, then $f x=g y=0, h z=1, T z=2$, hence we have

$$
\begin{aligned}
G^{2}(f 0, g 1, h 1) & =G^{2}(0,0,1)=1 \\
& <\frac{1}{3} \times 9=\frac{1}{3} G(0,0,2) G(0,0,2) \\
& =\frac{1}{3} G(f 0, S 1, T 1) G(R 0, g 1, T 1) \\
& \leq \frac{1}{3} M(0,1,1) .
\end{aligned}
$$

Case (15) If $x=z=0, y=2$, then $f x=h z=0, g y=1, S y=2$, hence we have

$$
\begin{aligned}
G^{2}(f 0, g 2, h 0) & =G^{2}(0,1,0)=1 \\
& =\frac{1}{3} \times 3=\frac{1}{3} G(0,2,0) G(0,1,0) \\
& =\frac{1}{3} G(R 0, S 2, h 0) G(f 0, g 2, T 0) \\
& \leq \frac{1}{3} M(0,2,0) .
\end{aligned}
$$

Case (16) If $x=0, y=2, z=1$, then $f x=0, g y=h z=1, R x=0, S y=T z=2$, hence we have

$$
\begin{aligned}
G^{2}(f 0, g 2, h 1) & =G^{2}(0,1,1)=1 \\
& <\frac{1}{3} \times 16=\frac{1}{3} G(0,2,1) G(0,1,2) \\
& =\frac{1}{3} G(R 0, S 2, h 1) G(f 0, g 2, T 1) \\
& \leq \frac{1}{3} M(0,2,1) .
\end{aligned}
$$

Case (17) If $x=0, y=z=2$, then $f x=h z=0, g y=1, S y=2$, hence we have

$$
\begin{aligned}
G^{2}(f 0, g 2, h 2) & =G^{2}(0,1,0)=1 \\
& =\frac{1}{3} \times 3=\frac{1}{3} G(0,2,0) G(0,1,1)
\end{aligned}
$$




$$
\begin{aligned}
& =\frac{1}{3} G(R 0, S 2, h 2) G(f 0, g 2, T 2) \\
& \leq \frac{1}{3} M(0,2,2) .
\end{aligned}
$$

Case (18) If $x=z=1, y=0$, then $f x=g y=0, h z=1, T z=2$, hence we have

$$
\begin{aligned}
G^{2}(f 1, g 0, h 1) & =G^{2}(0,0,1)=1 \\
& <\frac{1}{3} \times 12=\frac{1}{3} G(0,0,2) G(1,0,2) \\
& =\frac{1}{3} G(f 1, S 0, T 1) G(R 1, g 0, T 1) \\
& \leq \frac{1}{3} M(1,0,1) .
\end{aligned}
$$

Case (19) $x=y=z=1$, then $f x=g y=0, h z=1, T z=2$, hence we have

$$
\begin{aligned}
G^{2}(f 1, g 1, h 1) & =G^{2}(0,0,1)=1 \\
& <\frac{1}{3} \times 12=\frac{1}{3} G(0,0,2) G(1,0,2) \\
& =\frac{1}{3} G(f 1, S 1, T 1) G(R 1, g 1, T 1) \\
& \leq \frac{1}{3} M(1,1,1) .
\end{aligned}
$$

Case (20) If $x=1, y=2, z=0$, then $f x=h z=0, g y=1, S y=2$, hence we have

$$
\begin{aligned}
G^{2}(f 1, g 2, h 0) & =G^{2}(0,1,0)=1 \\
& <\frac{1}{3} \times 4=\frac{1}{3} G(1,2,0) G(0,1,0) \\
& =\frac{1}{3} G(R 1, S 2, h 0) G(f 1, g 2, T 0) \\
& \leq \frac{1}{3} M(1,2,0) .
\end{aligned}
$$

Case (21) If $x=z=1, y=2$, then $f x=0, g y=h z=1, T z=2$, hence we have

$$
\begin{aligned}
\psi(G(f x, g y, h z)) & =3 G(0,1,1)=3 \\
& <\frac{11}{4} \times 2=\frac{11}{4} G(1,2,2) \\
& =\frac{11}{4} G(h z, T z, T z) \leq \frac{11}{4} M(x, y, z) \\
& =\psi(M(x, y, z))-\phi(M(x, y, z)) .
\end{aligned}
$$

Case (22) If $x=1, y=z=2$, then $f x=h z=0, g y=1, S y=2$, hence we have

$$
\begin{aligned}
\psi(G(f x, g y, h z)) & =3 G(0,1,0)=3 \\
& <\frac{11}{4} \times 2=\frac{11}{4} G(1,2,2)
\end{aligned}
$$




$$
\begin{aligned}
& =\frac{11}{4} G(g y, S y, S y) \leq \frac{11}{4} M(x, y, z) \\
& =\psi(M(x, y, z))-\phi(M(x, y, z)) .
\end{aligned}
$$

Case (23) If $x=2, y=0, z=1$, then $f x=g y=0, h z=1, T z=2$, hence we have

$$
\begin{aligned}
\psi(G(f x, g y, h z)) & =3 G(0,0,1)=3 \\
& <\frac{11}{4} \times 2=\frac{11}{4} G(1,2,2) \\
& =\frac{11}{4} G(h z, T z, T z) \leq \frac{11}{4} M(x, y, z) \\
& =\psi(M(x, y, z))-\phi(M(x, y, z)) .
\end{aligned}
$$

Case (24) If $x=2, y=z=1$, then $f x=g y=0, h z=1, T z=2$, hence we have

$$
\begin{aligned}
\psi(G(f x, g y, h z)) & =3 G(0,0,1)=3 \\
& <\frac{11}{4} \times 2=\frac{11}{4} G(1,2,2) \\
& =\frac{11}{4} G(h z, T z, T z) \leq \frac{11}{4} M(x, y, z) \\
& =\psi(M(x, y, z))-\phi(M(x, y, z)) .
\end{aligned}
$$

Case (25) $x=y=2, z=0$, then $f x=h z=0, g y=1, S y=2$, hence we have

$$
\begin{aligned}
\psi(G(f x, g y, h z)) & =3 G(0,1,0)=3 \\
& <\frac{11}{4} \times 2=\frac{11}{4} G(1,2,2) \\
& =\frac{11}{4} G(g y, S y, S y) \leq \frac{11}{4} M(x, y, z) \\
& =\psi(M(x, y, z))-\phi(M(x, y, z)) .
\end{aligned}
$$

Case (26) $x=y=2, z=1$, then $f x=0, g y=h z=1, T z=2$, hence we have

$$
\begin{aligned}
\psi(G(f x, g y, h z)) & =3 G(0,1,1)=3 \\
& <\frac{11}{4} \times 2=\frac{11}{4} G(1,2,2) \\
& =\frac{11}{4} G(h z, T z, T z) \leq \frac{11}{4} M(x, y, z) \\
& =\psi(M(x, y, z))-\phi(M(x, y, z)) .
\end{aligned}
$$

Case (27) If $x=y=z=2$, then $f x=h z=0, g y=1, S y=2$, hence we have

$$
\begin{aligned}
\psi(G(f x, g y, h z)) & =3 G(0,1,0)=3 \\
& <\frac{11}{4} \times 2=\frac{11}{4} G(1,2,2)
\end{aligned}
$$




$$
\begin{aligned}
& =\frac{11}{4} G(g y, S y, S y) \leq \frac{11}{4} M(x, y, z) \\
& =\psi(M(x, y, z))-\phi(M(x, y, z)) .
\end{aligned}
$$

Hence, all of the conditions of Theorem 2.1 are satisfied. Moreover, 0 is the unique common fixed point of $f, g, h, R, S$, and $T$.

Corollary 2.1 Let $(X, G)$ be a G-metric space. Suppose mappings $f, g, h, R, S, T: X \rightarrow X$ satisfying the following conditions:

$$
\begin{aligned}
G^{2}(f x, g y, h z) \leq & a_{1} G(R x, S y, T z) G(f x, R x, R x)+a_{2} G(g y, S y, S y) G(h z, T z, T z) \\
& +a_{3} G(f x, S y, T z) G(R x, g y, T z)+a_{4} G(R x, S y, h z) G(f x, g y, T z) \\
& +a_{5} G(f x, S y, h z) G(R x, g y, h z)
\end{aligned}
$$

for all $x, y, z \in X$. Here $a_{i} \geq 0(i=1,2,3,4,5)$ and $0 \leq a_{1}+a_{2}+a_{3}+a_{4}+a_{5}<1$. If one of the following conditions is satisfied, then the pairs $(f, R),(g, S)$, and $(h, T)$ have a common point of coincidence in $X$.

(i) The subspace $R X$ is closed in $X, f X \subseteq S X, g X \subseteq T X$, and the two pairs of $(f, R)$ and $(g, S)$ satisfy the common (E.A) property.

(ii) The subspace $S X$ is closed in $X, g X \subseteq T X, h X \subseteq R X$, and the two pairs of $(g, S)$ and $(h, T)$ satisfy the common (E.A) property.

(iii) The subspace $T X$ is closed in $X, f X \subseteq S X, h X \subseteq R X$, and the two pairs of $(f, R)$ and $(h, T)$ satisfy the common (E.A) property.

Further, if the pairs $(f, R),(g, S)$, and $(h, T)$ are weakly compatible, then $f, g, h, R, S$, and $T$ have a unique common fixed point in $X$.

Proof Suppose that

$$
M(x, y, z)=\max \left\{\begin{array}{l}
G(R x, S y, T z) G(f x, R x, R x), \\
G(g y, S y, S y) G(h z, T z, T z), \\
G(f x, S y, T z) G(R x, g y, T z), \\
G(R x, S y, h z) G(f x, g y, T z), \\
G(f x, S y, h z) G(R x, g y, h z)
\end{array}\right\} .
$$

Then

$$
\begin{aligned}
a_{1} G( & (R x, S y, T z) G(f x, R x, R x)+a_{2} G(g y, S y, S y) G(h z, T z, T z) \\
& +a_{3} G(f x, S y, T z) G(R x, g y, T z)+a_{4} G(R x, S y, h z) G(f x, g y, T z) \\
& +a_{5} G(f x, S y, h z) G(R x, g y, h z) \\
\leq & \left(a_{1}+a_{2}+a_{3}+a_{4}+a_{5}\right) M(x, y, z) .
\end{aligned}
$$

So, if the condition (2.2) holds, then $G^{2}(f x, g y, h z) \leq\left(a_{1}+a_{2}+a_{3}+a_{4}+a_{5}\right) M(x, y, z)$. Taking $k=a_{1}+a_{2}+a_{3}+a_{4}+a_{5}$ in Theorem 2.1, the conclusion of Corollary 2.1 can be obtained from Theorem 2.1, since $0 \leq a_{1}+a_{2}+a_{3}+a_{4}+a_{5}<1$. 


\section{Competing interests}

The authors declare that they have no competing interests.

\section{Authors' contributions}

The authors contributed equally to the writing of the present article. They also read and approved the final manuscript.

\section{Author details}

${ }^{1}$ Department of Mathematics, Institute of Applied Mathematics, Hangzhou Normal University, Hangzhou, Zhejiang 310036, China. ${ }^{2}$ College of Statistics and Mathematics, Yunnan University of Finance and Economics, Kunming, Yunnan 650221, P.R. China.

\section{Acknowledgements}

The present studies are supported by the National Natural Science Foundation of China $(11071169,11361070)$, the Natural Science Foundation of Zhejiang Province (Y61 10287, LY12A01030), the Natural Science Foundation of Shandong Province (ZR2013AL015) and the Innovation Foundation of Graduate Student of Hangzhou Normal University.

\section{Received: 2 April 2014 Accepted: 10 November 2014 Published: 26 Nov 2014}

\section{References}

1. Mustafa, Z, Sims, B: A new approach to a generalized metric spaces. J. Nonlinear Convex Anal. 7, $289-297$ (2006)

2. Mustafa, Z, Obiedat, H, Awawdeh, F: Some fixed point theorems for mappings on complete G-metric space. Fixed Point Theory Appl. 2008, Article ID 189870 (2008). doi:10.1155/2008/189870

3. Mustafa, Z, Sims, B: Fixed point theorems for contractive mappings in complete G-metric spaces. Fixed Point Theory Appl. 2009, Article ID 917175 (2009)

4. Mustafa, Z, Shatanawi, W, Bataineh, M: Existence of fixed points results in G-metric spaces. Int. J. Math. Math. Sci. 2009, Article ID 283028 (2009)

5. Mustafa, Z, Khandagji, M, Shatanawi, W: Fixed point results on complete G-metric spaces. Studia Sci. Math. Hung. 48(3), 304-319 (2011)

6. Obiedat, H, Mustafa, Z: Fixed point results on a nonsymmetric G-metric spaces. Jordan J. Math. Stat. 3(2), 65-79 (2010)

7. Aydi, H, Shatanawi, W, Vetro, C: On generalized weakly G-contraction mapping in G-metric spaces. Comput. Math. Appl. 62(11), 4222-4229 (2011)

8. Aydi, H: A fixed point result involving a generalized weakly contractive condition in G-metric spaces. Bull. Math. Anal. Appl. 3(4), 180-188 (2011)

9. Gajić, L, Stojaković, M: On Ćirić generalization of mappings with a contractive iterate at a point in G-metric spaces. Appl. Math. Comput. 219(1), 435-441 (2012)

10. Zhou, SH, Gu, F: Some new fixed points in G-metric spaces. J. Hangzhou Norm. Univ., Nat. Sci. Ed. 11(1), 47-50 (2012)

11. Shatanawi, W: Fixed point theory for contractive mappings satisfying $\Phi$-maps in $G$-metric spaces. Fixed Point Theory Appl. 2010, Article ID 181650 (2010)

12. Chugh, R, Kadian, T, Rani, A, Rhoades, BE: Property P in G-metric spaces. Fixed Point Theory Appl. 2010, Article ID 401684 (2010)

13. Abbas, M, Rhoades, BE: Common fixed point results for noncommuting mappings without continuity in generalized metric spaces. Appl. Math. Comput. 215(1), 262-269 (2009)

14. Abbas, M, Nazir, T, Saadati, R: Common fixed point results for three maps in generalized metric space. Adv. Differ. Equ. 2011, Article ID 49 (2011)

15. Abbas, M, Nazir, T, Radenović, S: Some periodic point results in generalized metric spaces. Appl. Math. Comput. 217(8), 4094-4099 (2010)

16. Abbas, M, Khan, SH, Nazir, T: Common fixed points of R-weakly commuting maps in generalized metric spaces. Fixed Point Theory Appl. 2011, Article ID 784595 (2011)

17. Vats, RK, Kumar, S, Sihag, V: Some common fixed point theorems for compatible mappings of type $(A)$ in complete G-metric space. Adv. Fuzzy Math. 6(1), 27-38 (2011)

18. Abbas, M, Nazir, T, Vetro, P: Common fixed point results for three maps in G-metric spaces. Filomat 25(4), 1-17 (2011)

19. Gu, F: Common fixed point theorems for six mappings in generalized metric spaces. Abstr. Appl. Anal. 2012, Article ID 379212 (2012). doi:10.1155/2012/379212

20. Gu, F: Some new common coupled fixed point results in two generalized metric spaces. Fixed Point Theory Appl. 2013, Article ID 181 (2013). doi:10.1186/1687-1812-2013-181

21. Gu, F, Yang, Z: Some new common fixed point results for three pairs of mappings in generalized metric spaces. Fixed Point Theory Appl. 2013, Article ID 174 (2013). doi:10.1186/1687-1812-2013-174

22. Gu, F, Ye, H: Common fixed point theorems of Altman integral type mappings in G-metric spaces. Abstr. Appl. Anal. 2012, Article ID 630457 (2012). doi:10.1155/2012/630457

23. Ye, H, Gu, F: Common fixed point theorems for a class of twice power type contraction maps in G-metric spaces. Abstr. Appl. Anal. 2012, Article ID 736214 (2012)

24. Yin, Y, Gu, F: Common fixed point theorem about four mappings in G-metric spaces. J. Hangzhou Norm. Univ., Nat. Sci. Ed. 11(6), 511-515 (2012)

25. Ye, HQ, Lu, J, Gu, F: A new common fixed point theorem for noncompatible mappings of type $\left(A_{f}\right)$ in $G$-metric space. J. Hangzhou Norm. Univ., Nat. Sci. Ed. 12(1), 50-56 (2013)

26. Shen, YJ, Lu, J, Zheng, HH: Common fixed point theorem for converse commuting mappings in generalized metric spaces. J. Hangzhou Norm. Univ., Nat. Sci. Ed. 13(5), 542-547 (2014)

27. Hussain, N, Parvaneh, V, Hoseini Ghoncheh, SJ: Generalized contractive mappings and weakly $\alpha$-admissible pairs in G-metric spaces. Sci. World J. 2014, Article ID 941086 (2014)

28. Hussain, N, Parvaneh, V, Roshan, JR: Fixed point results for $G$ - $\alpha$-contractive maps with application to boundary value problems. Sci. World J. 2014, Article ID 585964 (2014)

29. Hussain, N, Roshan, JR, Parvaneh, V, Latif, A: A unification of G-metric, partial metric and b-metric spaces. Abstr. Appl. Anal. 2014, Article ID 180698 (2014) 
30. Tahat, N, Aydi, H, Karapınar, E, Shatanawi, W: Common fixed points for single-valued and multi-valued maps satisfying a generalized contraction in G-metric spaces. Fixed Point Theory Appl. 2012, Article ID 48 (2012). doi:10.1186/1687-1812-2012-48

31. Mustafa, Z: Common fixed points of weakly compatible mappings in G-metric spaces. Appl. Math. Sci. 6(92), 4589-4600 (2012)

32. Abbas, M, Nazir, T, Dorić, D: Common fixed point of mappings satisfying (E.A) property in generalized metric spaces. Appl. Math. Comput. 218(14), 7665-7670 (2012)

33. Mustafa, Z, Aydi, H, Karapınar, E: On common fixed points in G-metric spaces using (E.A) property. Comput. Math. Appl. 64(6), 1944-1956 (2012)

34. Long, W, Abbas, M, Nazir, T, Radenović, S: Common fixed point for two pairs of mappings satisfying (E.A) property in generalized metric spaces. Abstr. Appl. Anal. 2012, Article ID 394830 (2012). doi:10.1155/2012/394830

35. Gu, F, Yin, Y: Common fixed point for three pairs of self-maps satisfying common (E.A) property in generalized metric spaces. Abstr. Appl. Anal. 2013, Article ID 808092 (2013). doi:10.1155/2013/808092

36. Gu, F, Shatanawi, W: Common fixed point for generalized weakly G-contraction mappings satisfying common (E.A) property in G-metric spaces. Fixed Point Theory Appl. 2013, Article ID 48 (2013). doi:10.1186/1687-1812-2013-309

37. Jleli, M, Samet, B: Remarks on G-metric spaces and fixed point theorems. Fixed Point Theory Appl. 2012, Article ID 210 (2012). doi:10.1186/1687-1812-2012-210

38. Samet, B, Vetro, C, Vetro, F: Remarks on G-metric spaces. Int. J. Anal. 2013, Article ID 917158 (2013)

39. Mustafa, Z, Obiedat, H, Awawdeh, H: Some fixed point theorem for mappings on complete G-metric spaces. Fixed Point Theory Appl. 2008, Article ID 189870 (2008)

40. Karapınar, E, Agarval, R: Further remarks on G-metric spaces. Fixed Point Theory Appl. 2013, Article ID 154 (2013). doi:10.1186/1687-1812-2013-154

41. Jungck, G, Rhoades, BE: Fixed point for set valued functions without continuity. Indian J. Pure Appl. Math. 29, 227-238 (1998)

10.1186/1029-242X-2014-464

Cite this article as: Gu et al.: Common fixed point results under a new contractive condition without using

continuity. Journal of Inequalities and Applications 2014, 2014:464

\section{Submit your manuscript to a SpringerOpen ${ }^{\ominus}$ journal and benefit from:}

- Convenient online submission

- Rigorous peer review

- Immediate publication on acceptance

- Open access: articles freely available online

- High visibility within the field

- Retaining the copyright to your article

Submit your next manuscript at $>$ springeropen.com 